\section{Quantum optics with}

\section{gamma radiation}

Romain Coussement ${ }^{1,2}$, Rustem Shahkmouratov ${ }^{1,3}$, Gerda Neyens ${ }^{I}$ and Jos Odeurs ${ }^{1}$

${ }^{1}$ Instituut voor Kern-en Stralingsfysica, Katholieke Universiteit Leuven, Celestijnenlaan 200 D, B-3001 Leuven, Belgium

${ }^{2}$ Optique Nonlinéaire Théorique, Université Libre de Bruxelles, CP 231, Bld du Triomphe, B-1050 Bruxelles, Belgium

${ }^{3}$ Kazan Physico-Technical Institute of Russian Academy of

Sciences, 10/7 Sibirsky trakt, Kazan 420029, Russia

C AN one slow down a gamma photon to a group velocity of a few $\mathrm{m} / \mathrm{s}$, or can one stop it in a piece of material only a few micron thick and release it? Can one, on command, induce transparency of a nuclear resonant absorber for gamma radiation and make a gate? Can one change the index of refraction for gamma rays in such a way that one could think of optical devices for gamma radiation such as mirrors, cavities etc?

Such questions would all sound like science fiction if we did not know that such effects were first predicted by theoretical quantum optics and are observed experimentally with optical photons interacting with atoms [1-4]. Since gamma radiation and optical radiation are of the same electromagnetic nature, we can ask seriously why we could not observe the same effects when gamma radiation interacts with nuclear matter. These are the questions that one tries to answer in the field of "quantum nucleonics", sometimes also called "gamma optics".

There are obviously some important differences between quantum optics (interaction of photons with atomic electrons) and gamma optics (interaction of gamma photons with atomic nuclei). In particular for the investigation of the former the basic ingredient is the use of laser light (called the 'driving' laser) for inducing coherence and interference effects in atomic systems with three or more levels (Box 1: the basic principles of coherence and interference).

The exploration of coherence with gamma radiation has been hindered by the fact that one does not have a driving gamma ray laser. Furthermore, gamma radiation is emitted by a radioactive source and there is no phase coherence between photons as is the case for photons emitted by a laser in the optical domain. But this restriction can be circumvented to a large extent by the use of mixed level doublets in the nucleus (Box 2). We will show that the study of coherence and interference with gamma radiation has some unique features compared with the optical case.

First one should note that the gamma photons are emitted and detected as single photons. This is a restriction, but it also provides a unique opportunity to see interference effects using a single photon. Optical single photon experiments require sophisticated equipment (which is available but needs special care and skills), while with gamma radiation each experiment is a single photon experiment. It allows us to address some fundamental questions about the quantum mechanical, dual nature of the single photon.

Another interesting feature is that one can easily choose gamma rays emitted from a nuclear isomer. Because of the long lifetime of the isomeric state, its line width is extremely small and the coherence length of the emitted photon is very long. For the gamma rays of $14.4 \mathrm{keV}$ emitted by the excited ${ }^{57 m}$ Fe state ( $\tau=141 n s, \mathrm{I}=3 / 2$ ), the coherence length is about $40 \mathrm{~m}$. For the 6.2 $\mathrm{keV}$ gamma radiation from ${ }^{181 \mathrm{~m}} \mathrm{Ta}$, having a lifetime of $8.73 \mu \mathrm{s}$, the coherence length is $4 \mathrm{~km}$. The appreciable coherence length of these gamma photons allows us to observe the interference between the transition amplitudes from two paths of the single photon, passing through two different samples. In one path the photon interacts with nuclei of a reference sample and in the other path it interacts with the nuclei of a sample under investigation. The distance between the samples can be as large as the coherence length of the photon. Interference of these two transition amplitudes provides spectroscopic information about the hyperfine interaction of the nuclei in the investigated sample, provided the hyperfine spectrum of the nuclei in the reference sample is known. Such interference phenomena have been explored using synchrotron radiation [5] and are nowadays used as a tool for solid state physics studies.

Because of their very high energy, the gamma photons have very short wavelengths: of the order of an Angström or a fraction of it. Consequently, it becomes possible to investi-

Since the discovery of optical lasers, the scientific community has been challenged to realise a gamma ray laser. gate crystalline structures using nuclear Bragg scattering. Methods based on this feature, such as nuclear emission [6] and absorption holography [7], have been explored recently.

Having photons with a very long coherence length and a very small wavelength allows us to conceive an interferometer that is very sensitive to small changes in optical length. The idea has not yet been explored and one of the possible applications may be the detection of the gravity red shift with a much higher precision [8].

Because nuclear cross sections are many orders-of-magnitude smaller than the atomic ones, nuclear gamma optics experiments require the use of high density targets, which means the use of

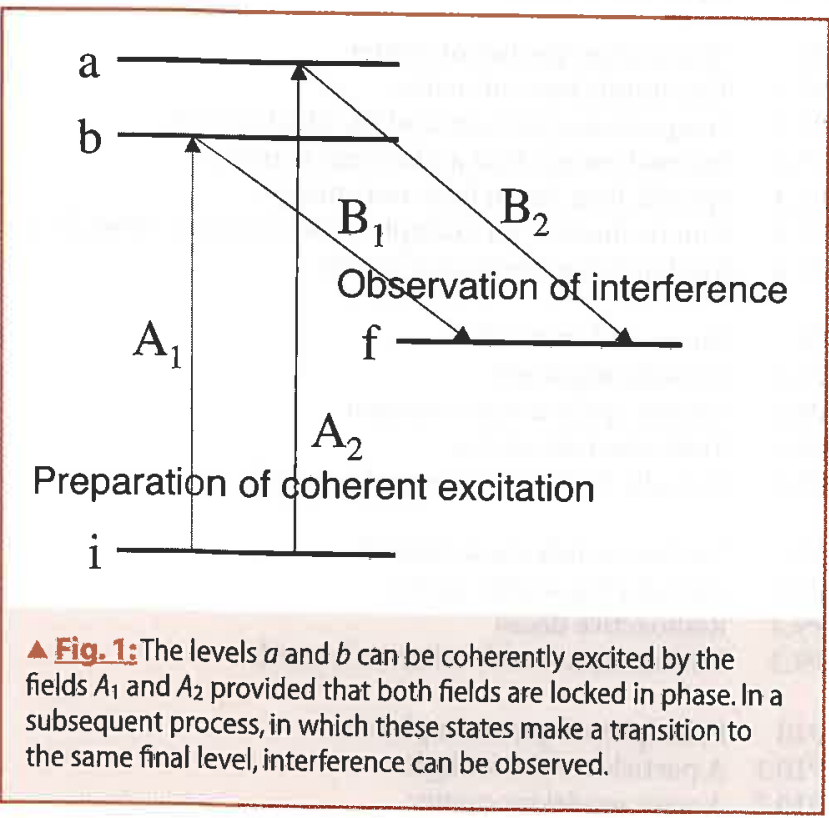


solid materials. As the nuclear recoil energy induced by the gamma decay is very large (because of the large gamma ray energy) and because the recoil energy depends on the spectrum of phonons created in the lattice, the gamma ray emitted from a nucleus embedded in a solid has a large statistical energy distribution. To allow experiments with narrow gamma lines it is necessary to use solids in which this large statistical energy variation is reduced. This is a major restriction on the gamma transitions available for gamma optics research, as only Mössbauer isotopes fulfill this condition. For such isotopes the recoil momentum is transferred to the lattice as a whole and the gamma decay is registered as a 'recoilless' decay, giving rise to narrow gamma ray lines. One could lift this restriction by using dilute gases or mono-energetic beams of nuclear isomers [9]. The recoil energies would then be the same for all events, and averaging all transition amplitudes over a large ensemble of events would not lead to the cancellation of the interference term. However the smallness of the densities together with the small cross sections put such experiments out of reach with the present means of producing nuclear isomers.

\section{The gamma ray laser}

Since the discovery of optical lasers, the scientific community has been interested and challenged to realise a gamma ray laser (see for example [12]). A gamma ray laser would offer many applications because of the short wavelength and because of the high power density. Despite the considerable efforts of many groups, there still exists no idea of how to build such a device using present technology and our available knowledge of laser, nuclear and atomic physics.

The main problem is the realisation of population inversion. Because of the small cross sections for nuclear photo-absorption, an adequate pump mechanism does not exist. Because the pump efficiency is very weak, while the losses due to electronic absorption processes are important, one needs a tremendous energy input. Under extreme pump irradiation, the non-resonant power input would be so large that the sample would be destroyed by melting or evaporation before lasing could occur. As a result we are faced by the so called gamma laser dilemma [13] in that one needs a solid with a high recoilless fraction and that the condition for recoilless fraction will be destroyed by the required pump power. To try to circumvent this dilemma, one follows two avenues. They have in common that one will store energy in long-lived isomers and find a mechanism to release it on command. Storing large amounts of energy in nuclear isomers is not the real challenge. One can produce these long-lived isomers in nuclear reactors or with accelerator beams and separate them from other types of material by chemical and/or physical means. The technology is available in principle or at least there is enough knowledge available for it to be developed. The problem is the release of the stored energy 'on command' and in a very short time. It is on this point that the two approaches diverge conceptually.

\section{Energy conversion}

One of the roads followed is to pump a long-lived nuclear isomer into an excited nuclear state via low energy X-ray irradiation. Subsequently this excited state decays via the emission of gamma rays, representing a multiple of the input energy (see figure 3). In this scenario the long-lived isomeric state could act as a nuclear battery, in which energy is stored.

A proof of principle for releasing the stored energy has been demonstrated using a K-isomer as the storage level [14]. From the point of view of nuclear models the result is surprising. Indeed,

\section{Box 1}

\section{Coherence and Interference}

A quantum transition from state $i$ to state $f$ can be described by an amplitude $A=A_{i j}^{\prime} \exp (i \varphi)$, where $\varphi$ stands for the phase of the field inducing this transition and $A_{\text {if }}^{\prime}$ is the complex transition matrix element, which depends on the relative phases of the initial and final states. In most processes all the phases are cancelled since the observable is just a transition probability $P=|A|^{2}$. However, if the experiment is designed in order to observe a process with two quantum paths, one must first sum the amplitudes and then square the norm. This procedure will produce interference terms in which the phase factors play an important role:

$$
P=\left|A_{1}+A_{2}\right|^{2}=\left|A_{1}\right|^{2}+\left|A_{2}\right|^{2}+\left[A_{1}^{\prime} A_{2}^{\prime *} e^{i\left(\varphi_{1}-\varphi_{2}\right)}+A_{1}^{\prime *} A_{2}^{\prime} e^{-i\left(\varphi_{1}-\varphi_{2}\right)}\right]
$$

In an experiment a large number of events is observed. If all the phases are different from event to event then the experimentally observable is an average of all possible phase differences. The interference term cancels and the probability is just the sum of the partial probabilities $P_{i}=\left|A_{i}\right|^{2}$.

However one can design an experiment in which the interference term is not zero. Then the interference is constructive or destructive, depending on the sign of the phase difference $\varphi_{1}-\varphi_{2}$, if the latter is fixed for the ensemble or chain of events. This can be done if the transition is split in two paths due to the two-step processes, as shown in Fig. 1. In one step $\left(A_{1}\right.$ and $\left.A_{2}\right)$ the coherence between $a$ and $b$ states is prepared, if the phase difference of the $A_{1}$ and $A_{2}$ fields is fixed (which is the case of the phase locked fields or mutually correlated fields). In the second step ( $B_{1}$ and
$B_{2}$ ) the interference is observed. The arbitrary phase of the intermediate state does not affect the result because this state occurs with its complex conjugate in the transition amplitude from state $i$ to state $f$, i.e., $\left(A_{1}^{\prime}\right)_{i b}\left(B_{1}^{\prime}\right)_{b f}$. When calculating the transition probability of two quantum paths, each following a two-step process (Fig. 1), the phase factors of the initial and the final states cancel, provided that both paths start and end at the same initial and final states. Coherent preparation is mostly called coherent excitation and the interferences are observed in the subsequent step. However the role of the preparation and observation step may be reversed. In that case the interferences can be observed in the first step, provided that the experiment is constructed such that the observed transition in the furst step is conditioned by the second step.

In short, the rule for observing interference in quantum transitions is that the observed transition can pass via two indistinguishable quantum paths, which have a common initial and a common final state, and that the radiation fields in the preparation step are phase locked.

While in quantum optics the phase locking of the laser fields became a routine, in case of gamma radiation, where single photon processes are to be considered, this problem needs special care. To be coherent the first step of excitation must go to closely spaced nuclear levels. These levels are to be close enough in energy that a single photon can induce the transition to the two states, despite its sharp frequency distribution. In box 2 we explain how to produce such closely-spaced nuclear levels and later in this article we demonstrate that they fulfill all the conditions to be coherently excited by a single gamma photon. 
the $\mathrm{K}$-isomeric state has a long lifetime because the $\mathrm{K}$-selection rule hinders its decay into nuclear states at lower energy as this involves a large change in the projection of the angular momentum [15]. It comes as a surprise that the transitions to higher energy states in this ground state band are then less affected by this hindrance. It is certainly an interesting phenomenon for nuclear spectroscopists. However because of the weak coupling of the X-ray pump with the possible lasing level, this is not yet an efficient mechanism for producing a gamma ray laser.

\section{Lasing without inversion}

A prime condition for gain with stimulated emission is population inversion, or explicitly, the number of excited state nuclei from which lasing has to occur, exceeds the number of ground state nuclei. This condition requires that one must be able to perform a nuclear isomeric separation in order to obtain nearly pure isomeric material. Such a technology could be developed in the future, for example using the laser ionisation method for producing isomeric enrichment, the separation being based on the difference in the hyperfine structure [16].

Even if a solid material could be prepared with most of the nuclei in the ground state as well as a large number in some longlived isomeric state, without having an inverted system, lasing might still be realised. To obtain lasing from such a system, the concept of "lasing without inversion" as introduced in quantum optics, could be translated to gamma radiation. In the optical range the effect of "lasing without inversion" has been demonstrated experimentally [4] and it was pointed out that the main new application will be the realization of lasers at very high frequency, for example UV, X-ray or even a gamma ray laser. The main point here is that one can create coherence in a three level system in such a way that absorption of the lasing frequency is cancelled by destructive interference while the emission, and in particular the stimulated emission, is not [18].

In optics one can coherently excite two atomic levels by irradiating the atoms with two lasers of different frequency (color) but coupled in phase. Such phase-locked, two-frequency laser light is called 'bichromatic light' and can easily be obtained from one laser using frequency doubling or dividing crystals.

One does not have an equivalent tool in gamma optics. Therefore one is restricted to excitations of two 'nuclear hyperfine levels' which can be excited with a single gamma photon despite its small natural line width. Two conditions need to be fulfilled for this. One, the energy difference between these two levels must be less than the natural line width of the photon. This condition can easily be fulfilled by the method of nuclear level mixing, also sometimes called nuclear level anticrossing (see box 2). Secondly, the selection rules, which apply to transitions between nuclear hyperfine levels, need to be allowed in both paths. This condition is automatically fulfilled by the nature of the mixed levels produced via nuclear level mixing: the doublet consists of an in-phase and an anti-phase superposition of $\mathbf{m}$-quantum states. The selection rules, also taking into account the polarization of the photon, allow for example the transition to the angular momentum component $\mathrm{m}$ (which occurs in both mixed levels). Excitation of both levels of the doublet can then be achieved with a single photon.

In order to observe interference effects in this absorption process, another condition needs to be fulfilled, namely the two interfering quantum paths need to be fully coherent (see box 1). The coherence is created by the connection of the two states of the doublet with a single fourth quantum state with some resonant or quasi-resonant radiation (called the driving field). Such radia-

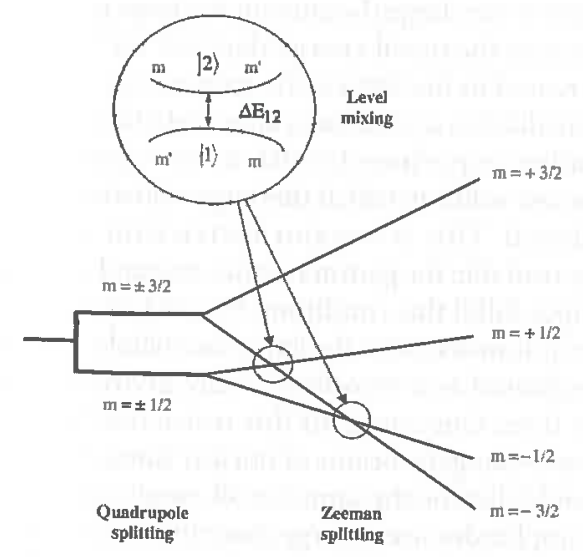

Fig. 2: Nuclear hyperfine levels as a function of the magnetic field, for the $I=3 / 2$ isomeric state in ${ }^{57} \mathrm{Fe}$ subjected to an electric field gradient and a static magnetic field. If the symmetry axes of both interactions are parallel, crossing of hyperfine levels occurs at particular values of the magnetic field (small circles). If the axial symmetry is broken by slightly misaligning the magnetic field with respect to the electric field gradient symmetry axis, then the hyperfine levels are mixed (enlarged part around the crossing).

tion can for example be induced by a radio frequency (rf) magnetic field (also called a Nuclear Magnetic Resonance, NMR, field) [18]. In that case the fourth level must be a member of the same hyperfine manifold. It can also be induced by the coupling between the atomic and nuclear spins, via the excitation of the atomic state. In that case the radiation can be optical [19].

Another problem that remains is related to the weak coupling of the nuclei with the gamma radiation. When choosing nuclei with a very long lifetime, there is enough time to produce large quantities of the isotope, to make isomeric enrichment and to store it before triggering the release of all stored energy by turning on the driving radiation. However, there is a price to pay. As the isomeric state has a long lifetime, the coupling of the gamma radiation to the nuclei is weak. Thus the cross section for stimulated emission is small and the losses from electronic absorption can not be compensated by the gain from stimulated emission.

There could be a way out of this problem if the gamma radiation could be coupled to a collective ensemble of excited nuclei to form a state similar to a Bose Einstein condensate of atoms. In such a condensate, the probability for stimulated emission is multiplied by the number of excited nuclei in the collective ensemble. Now that Bose Einstein condensates have been produced in atomic systems, one might in the future think of ways to create a nuclear collective coherent state and thus pave the way for a gamma ray laser.

\section{Nuclear level mixing induced transparency}

Consider an ensemble of nuclei in their nuclear ground state, and that these nuclei have an excited state that is isomeric. Suppose the ensemble is submitted to the necessary conditions for creating a level mixed doublet in the isomeric state. As explained in box 1 , interference in such a system can occur in the decay from such coherently excited, level mixed states, provided decay to a particular final state is observed. This final state could be a particular member of the ground state manifold, even the same state from 
which the mixed states were populated. Indeed in such a scheme there are two paths which start and end on the same state so that the quantum phase factors cancel. However, one must notice that once the doublet states are excited, spontaneous decay will happen not only to one but to all members of the ground state hyperfine manifold. One can demonstrate that in the case of a spontaneous decay in a $4 \pi$ geometry the sum of the interference terms will cancel. In order to observe interference in the decay process, decay to a particular final state needs to be observed which means that the $4 \pi$ symmetry must be broken.

For a large ensemble of nuclei, the $4 \pi$ symmetry of the re-emission can be broken to some extent because of collective decay in some directions. It is well known from theories and experiments on elastic dynamical diffraction that the re-emission in the forward direction is enhanced.

The enhancement of the radiative decay in the forward direction can be understood as a collective effect. When a photon hits the sample, it hits all the resonant nuclei in their ground state. In the quantum mechanical picture of a photon being a particle, we would say that one of these nuclei may be excited with a certain probability. In the quantum mechanical picture of the photon as a wave, each resonant nucleus can be considered as a superposition of the "ground state plus a photon" and the "excited state without a photon". In this way the photon is not absorbed by one of the nuclei but by the 'collective ensemble' of nuclei. The reemission occurs then from this collective excitation, which is called an 'exciton'. The radiative decay from such an exciton is very special because each nucleus will give an amplitude for the emitted photon. The phases at a point of the detector will depend on the difference in optical length between two quantum paths (originating from the decay of two different nuclei). In the forward direction these lengths are equal, while in a single crystal they are proportional to an integer number of wavelengths if scattering in the Bragg directions is observed. In these directions all the individual amplitudes add up coherently and consequently the emission probability is enhanced by a factor equal to the number of nuclei involved.

We can thus conclude that for a thick absorber the radiative deexcitation in the forward direction toward the initial state is favoured over the other decay modes, including the non radiative

Fig. 3: The pump
scheme, using a "nuclear
energy battery" as a way
towards a gamma-ray laser.
The initial state is a long-
lived state at high spin and
excitation energy (in which
all nuclei are produced by
some means). The state is
isomeric because decay to
the lower levels is forbidden
by K-selection rules. The
energy from this 'battery' is
released by pumping it into
an intermediate 'K-mixed'
state, which subsequently
decays via a cascade of
gamma transitions. K-
mixing is here the crucial
ingredient to open the
decay channel.

decay modes. The enhancement in the forward direction strongly depends on the thickness of the absorber. In such conditions we can expect that interferences can be observed because, in the case of a strong enhancement, the process of excitation and subsequent deexcitation is reduced to the two dominant paths. These two paths are coherent because they both start and end on the same level. These interferences can, in principle, lead to more or to less absorption. Interferences will be observed for gamma photons which can excite both levels of the hyperfine doublet. The requirement is that the bandwidth of the incoming gamma photon must be about the same as the energy splitting of the doublet states. With the technique of nuclear level mixing (box 2), the energy splitting of two nuclear hyperfine levels can be fine tuned. An experiment was performed using the Mössbauer absorption of the $14.4 \mathrm{keV}$ radiation by the ${ }^{57}$ Fe nuclei in a single crystal absorber of $\mathrm{FeCO}_{3}$. This material has a hexagonal close packed lattice structure, thus inducing a quadrupole splitting of the

\section{$B 0 \times 2$}

\section{Crossing and mixing of nuclear hyperfine levels}

Consider nuclei subjected to static electromagnetic fields. The interaction of the nuclear magnetic moment with a magnetic field induces a Zeeman splitting of the nuclear m-quantum states. The interaction of the nuclear quadrupole moment with an electric field gradient gives rise to a quadrupole splitting. If both interactions are applied simultaneously and with their symmetry axis parallel, one obtains nuclear hyperfine levels as shown in figure 2. Because the quadrupole splitting is proportional to $\mathrm{m}^{2}$, while the Zeeman splitting is proportional to $\mathrm{m}$, the crossing of two quantum levels occurs at particular magnetic fields, called crossing fields, $H_{c}$. The ratio of the magnetic and quadrupole interaction frequencies determines the magnetic field at which the crossing will occur [10].

When the axial symmetry is broken slightly, because of a small misalignment of the magnetic field with respect to the symmetry axis of the electric field gradient, one can show that its effect on the quantum levels and their wave functions is negligible, except near the magnetic fields $H_{c}$ where level crossing occurs. At these fields, the degeneracy of m-quantum states is lifted due to the small degree of symmetry breaking, which can be demonstrated by quasi-degenerate perturbation theory [10]. Anti-crossing of nuclear hyperfine levels occurs and the wave functions are mixed in coherent superpositions of the wave functions belonging to the crossing states. At the crossing field, the mixed wave functions form exactly an in-phase and an anti-phase superposition of $m$ and $m^{i}$ (inset of figure 2 ):

$$
\begin{aligned}
& |1\rangle=1 \sqrt{ } 2\left\{|m\rangle+\left|m^{\prime}\right\rangle\right\} \\
& |2\rangle=1 \sqrt{ } 2\left\{\left|m^{\prime}\right\rangle-|m\rangle\right\}
\end{aligned}
$$

The minimum energy difference between the levels occurs at the crossing field and is dependent on the strength of the symmetry breaking term, which is determined by the misalignment angle between the applied interactions. Thus we can adjust the energy difference between the two levels.

Such mixing nuclear hyperfine states have been used in several fields of physics. For example as a tool to investigate the structure of solids using synchrotron radiation [5] or as a tool to study the structure of exotic nuclear states using their anisotropic radioactive decay [11]. They may also provide a step towards a gamma ray laser, as discussed later in this article. 
nuclear levels in ${ }^{57} \mathrm{Fe}$. Below $40 \mathrm{~K}$, the material becomes magnetic as well, thus allowing the fulfilment of the level mixing conditions (figure 2). In a Mössbauer absorption measurement, the intensity in the Mössbauer line corresponding to the absorption into the level mixed doublet, does not correspond to the sum of the intensities of the individual transitions [20]. This is a clear signature for the presence of interference effects in nuclear gamma ray absorption towards level mixed nuclear hyperfine levels. Moreover the effect of coherence is demonstrated for single photon events.

\section{Slow group velocity for gamma radiation?}

From classical electromagnetism it is well known that an electromagnetic pulse can propagate through a resonant medium with a group velocity that can be much slower than the phase velocity or the velocity of light [21]. The effect derives from the fact that near a resonance the index of refraction changes drastically. Therefore, each frequency component of the pulse will have another velocity, resulting in a slower propagation of the pulse shape through the medium. In a two level system it is difficult to observe the effect because, at resonance, there is strong resonant absorption and, consequently, the pulse will be just absorbed and not transmitted to the detector.

In a three level system, however, the absorption is cancelled in the energy (or frequency) region about halfway between the doublet levels. Exactly in that region, the index of refraction changes enormously [ 4$]$ and consequently the group velocity can be much slower than the velocity of light. From the transparency that was observed in the Mössbauer experiment [20] one can estimate that the propagation velocity of the gamma photon is reduced to about $1 \mathrm{~km} / \mathrm{s}$.

\section{Conclusions}

Coherence and interference effects in the interaction of light with matter led to very interesting and somewhat unexpected results in the field of quantum optics, such as gain without inversion, electromagnetically induced transparency, changes in index of refraction etc. [4]. In the challenging quest for a laser with gamma radiation these phenomena have been suggested as possible solutions to the gamma-ray laser dilemma. For that, a translation of the optical phenomena into the field of gamma optics, the interaction of gamma radiation with nuclei, has to be performed. A rich field of research related to coherence and interference in gamma radiation has evolved out of this.

Because of the single photon character of nuclear events, the conditions for coherence and interference need to be realized in a very different way. For example the bichromatic driving field that is used in optics, is replaced by a single photon and in the nuclear three level system a particular hyperfine doublet, as obtained from anticrossing of two nuclear hyperfine levels (Box 2) needs to be used to allow coherent excitation (box 1).

The concepts of coherence and interference were applied to nuclear resonant scattering of synchrotron radiation and a new nuclear interferometer was designed in which one observes the interference between two nuclear resonant scattering amplitudes, one from a reference sample and one from a sample under investigation. From these experiments the hyperfine splitting of the investigated sample can be deduced. This technique has opened a new field of application in the investigation of material properties using nuclear radiation.

The study of coherence phenomena in nuclear radiation led to useful applications also in other fields. For example in nuclear physics the principle of level mixing resonances (LMR) is used for the determination of spins, magnetic and quadrupole moments of exotic nuclei that are produced at a few accelerators in the world.

Finally, lasing and gain without inversion cannot be achieved in nuclear transtions so far, because an adequate pump mechanism is still missing. On the contrary, nuclear level mixing induced transparency has been observed experimentally and can be considered as the nuclear equivalent of optical, electromagnetically induced transparancy. Future investigations in this rich field of research, at the border of nuclear physics, quantum optics, solid state physics and laser physics, will certainly reveal interesting new phenomena and applications for the future.

\section{Acknowledgements}

The authors are very grateful to P. Mandel (U.L.B., Belgium), O. Kocharovskaya and Y. Rostovtsev (Texas A\&M, USA) for fruitful exchange of ideas on this subject.

\section{References}

[1] For recent achievements in this domain of quantum optics, see, for example, S. E. Harris, Physics Today 50, No 7, 36 (1997); M. Xiao, H. Wang, and D. Goorskey, Optics \& Photonics News, p.44, September 2002.

[2] O. Kocharovskaya, Phys. Rep. 219, 175 (1992).

[3] M. O. Scully, Phys. Rep. 219, 191 (1992).

[4] C. Liu et al., Nature 409,490 (2001); D. F. Phillips et al., Phys. Rev. Lett. 86, 783 (2001); A. B. Matsko et al., Advances in Atomic, Molecular and Optical Physics 46, 191 (2001).

[5] R. Coussement et al., Phys. Rev. B 54, 16003 (1996); R. Coussement et al., Hyp. Int. 125, 113 (2000), R. Callens et al., Phys. Rev. B 65, 180404 (2002)

[6] J. Odeurs et al., Phys. Rev. B 60, 7140 (1999).

[7] P. Korecki et al., Phys. Rev. Lett. 79, 3518 (1997); Phys. Rev. B 59, 6139 (1999).

[8] H. Frauenfelder, “The Mössbauer effect”, Ed. Benjamin 1963, New York

[9] L. A. Rivlin, Hyp. Int. 107,57 (1997).

[10] R. Coussement et al., Hyp. Int. 23, 273 (1985)

[11] G. Neyens et al., Phys. Lett. B 1-2, 36 (1997)

[12] Proc. of the Int. Gamma Ray Laser conference (GARALAS'97), Hyp. Int. 107 (1997).

[13] G. C. Baldwin, J. C. Solem, and V.I. Goldanskii, Rev. Mod. Phys. 51, 4 (1981); G. C. Baldwin and J. C. Solem, Laser Phys. 5, 231, 326 (1995).

[14] C. B. Collins et al., Phys. Rev. Lett, 82, 695 (1999); J. J. Carroll et al., Hyp. Int. 135, 3 (2001); C. B. Collins et al., Hyp. Int. 135, 51 (2001).

[15] P. Walker and G. Dracoulis, Nature 399, 35 (1999)

[16] U. Köster et al., Nucl. Instr. and Meth. in Phys. Res. B 160, 528 (2000)

[17] E. S. Fry et al, Phys. Rev. Lett. 70, 3235 (1993); W. E. van der Veer et al, Phys. Rev. Lett. 70, 3243 (1993); J. Vanier et al, Phys. Rev. A 58, 2345 (1998); K. Yamamoto et al, Phys. Rev. A 58, 2460 (1998);

[18] R. Coussement et al., Phys. Rev. Lett. 71, 1824 (1993).

[19] R. N.Shakhmuratov et al., Opt. Comm. 179, 525 (2000); G. Kozyreff, et al., Phys. Rev. A 64, 013810 (2001).

[20] R. Coussement et al., Phys. Rev. Lett. 89, 107601 (2002).

[21] J.D. Jackson, "Classical Electrodynamics", 2nd ed. John Wiley and Sons, New York, 1975 